\title{
Effect of nursing guidelines on patients' outcomes regarding closed suction at intensive care unit.
}

\author{
Hanan Mohran Mousa ${ }^{1}$, Moggeda Mohamed ${ }^{2}$ \& Naglaa Ahmed Ahmed ${ }^{3}$. \\ 1- Nursing supervisor in Pediatric I.C.U. Assuit University, Egypt. \\ 2- Assist Professor of Critical Care and Emergency Nursing Department, Faculty of Nursing Assuit University \\ Egypt. \\ 3- Lecturer in Critical Care and emergency NursingDepartment, Faculty of Nursing Assuit University, Egypt.
}

\begin{abstract}
Introduction: Application of nursing guideline of closed suction system had a positive effection critically patient's outcomes, took shorter time and is more economical. Aim of study: was to evaluate the effect of nursing guidelines on patient's outcomes regarding closed suction at intensive care unit. Design: Quasi - experimental research design. Setting, the study was conducted in pediatric intensive care unit at Assuit University hospital. Sample purpose sample 60 critically ill patient's were included in the study. There are categorized into two groups connected with mechanical ventilation who used closed suction from both sex study and control group (30 in each). Tools: three tools developed by the research after review of literatures to assess the patient condition to form baseline data these tool comprised Tool I-patient assessment, tool II-suction assessment, and tool III-outcomes patient tool. Results: The results of the study showed that the effectiveness of nursing guidelines of closed suction system is positive and clear. There was a good improvement of patient's status, the findings of the current study revealed there was significant difference between study and control group regarding to respiratory infection (10) Verus (20), the findings of the current study revealed there was significant difference between study and control group regarding to mortality rate in the study $(66.7 \%)$ less than control group $(93.3 \%)$. Conclusion: application of nursing guideline of closed suction system had a positive effect in critically patients out comes. Recommendation Keep the nursing guideline of closed suction system available in all the intensive care units.
\end{abstract}

\section{Keywords: Acquired Hospital Infection Closed Suction System, Nursing \& Health Care, Secretion.}

\section{Introduction}

Tracheal suctioning is one of the critical nursing interventions to keep the airway patent by removing secretion via suctioning. This critical skill requires expertise with the knowledge to perform and facilitate a patient's effortless breathing pattern through effective secretion management. But this aspect of care is associated with the risk of injury therefore, the role of competent health care professionals (HCP) is vital in performing tracheal suctioning (Khimani1, et al., 2015, Elosama 2017).

Nowadays, two systems are available to perform ES: open suction system (OSS) and closed suction system (CSS). During open ES, the patient is first disconnected from the mechanical ventilator, if present; the airway is suctioned using a disposable sterile catheter connected to the vacuum system, and the patient is then re-connected to the ventilator During closed ES, the aspiration catheter is connected to the ventilator line and is a part of the ventilator circuit; it is not necessary to disconnect the patient from the ventilator during the procedure. Moreover, CSS needs special designed catheter which is more expensive and not easy to be available. Clinical studies have demonstrated that OSS can cause alveolar decruitment and transient hypoxemia. In Egypt, OSS is the most common used technique because its suction catheter is less cost and more available (Elsaman 2017).

Endotracheal intubation and the institution of invasive mechanical ventilation are resources which are widely used in the management of critically-ill patients, so as to provide sufficient gases exchange for those with some sort of respiratory insufficiency. However, these devices can cause detrimental effects - inflammation, infections, and traumatic lesions to the airways, which require preventive care. The appropriate management of the artificial airway has a direct impact on the patients' prognosis, including reduction of morbimortality, length of hospitalization, and hospital costs. (Frota et al., 2014).

Effective suctioning is an essential aspect of airway management in the intubated critically ill patients. They are unable to maintain a patent airway as glottic closure is compromised, preventing cough reflex, increasing secretions and also compromising their ability to clear endotracheal secretions). In a healthy patient, the action of ciliated cells in the airways, the local immune system, and the cough reflex are essential for the destruction and removal of microorganisms as well as clearing debris from the lungs. However, in the critically or acutely ill patient, these 
functions may be severely compromised, resulting in an excessive production of secretions, which may prove difficult to expectorate. Endotracheal and tracheostomy tubes form artificial airways, which bypass the normal physiological processes and inhibit the cough reflex .(Royal Children's Hospital 2012). This leaves the respiratory tract vulnerable to opportunistic infections, with an increased production of mucus and reduced secretion of pneumocytesand surfactant. An inability to expectorate this mucus, which is often thick and tenacious, is a common problem for the patient with a tracheostomy or endotracheal tube. Periodic suctioning is required in order to clear these secretions and prevent atelectasis or alveolar collapse. (Royal Children's Hospital 2012).

The two standard and well-known methods for suctioning are the open suction system and the closed suction system. The open suction system works by disconnecting the ventilator circuit and inserting a catheter. In closed suction system is done by using a catheter in an enclosed sheath attached to the inside of the tracheal without disconnected (Evans 2014, Haghighats 2015) One of the main goals of nursing is to provide evidence-based assistance in order to promote quality results for patients, families and the healthcare system. A procedure that deserves particular attention, given its direct relationship with the risk of infection, is the endotracheal aspiration (ETA) of intubated patients. (Frota et al., 2013)

The nurse monitors the patient's response to ventilation, intervenes to maintain oxygenation and ventilation and ensures that the patient's complex needs are met. Therefore, in order to provide a better comprehensive care to the mechanically ventilated patient and to reduce the cases of common complications of endotracheal suctioning, directed at removing secretions and, through this, promoting the maintenance of the airways' permeability, as well as optimizing ventilation and oxygenation. The procedure is essential for the stability of pulmonary function, as the presence of a ventilatory prosthesis interferes in the physiology of coughing and of the mucociliary system, which can inviabilize the adequate clearance of secretions from the tracheobronchial tree and cause stasis of this content (Sharma et al., 2014).

\section{Significance of the study}

Endotracheal suctioning is a necessary procedure practiced by nurse in intensive care unit to remove lung secretions the procedure lead to higher oxygenation levels and reduced breathing difficulties. The incidence of the suction closed suction system in $70 \%$ of hospitalized patient in pediatric intensive care unit and $30 \%$ open suction system of the patient in pediatric intensive care unit at Assiut University Hospital. Closed-suction catheter is widely used in the ICU due to favorable outcomes, including improved oxygenation, reduced hypoxia, positive end-expiratory pressure maintenance and subsequent decrease in atelectasis and decreased risk of nosocomial infection. Closed suction systems may be more effective for the ICU environment where ICU bed density is too high.

Operation definition

Closed suction system is a novel device-used to a separate liquids or semi solids from a patient's upper airways, which is integrated and connecting between the closed circuit from the ventilator and the breathing tube, endotracheal tube or the tracheostomy tube, ventilator support remains intact and optimized as no disconnection from the ventilator is necessary it also minimizes infection risk.

The outcomes for this meta-analysis is was evaluated the effect of nursing guideline on patient's outcomes regarding closed suction system at intensive care unit.

\section{Aim of the study \\ The present study was aim to}

Evaluate the effect of nursing guidelines on patient's outcomes regarding closed suction system at intensive care unit.

\section{Patients \& Method \\ Research design}

Aquasi experimental research design was adopted to conduct this study

\section{Setting}

The study was conducted in pediatric intensive care unit at Assiut University Hospital from (1/4/2017 to 31/12/2018)

\section{Sampling}

Purposuive sample consisted of 60 patients (male and female) in the previous setting were not randomly included in the study, their number was divided equally in to two groups ( 30 control and 30 study)

\section{Criteria of the sample}

\section{Inclusion criteria}

All patients admitted in ICU and attached with mechanical ventilation more than 7 days.

Exclusion criteria

Cancer patient's, burn patient's, and COPD .

Hypothesis

Patient's who will be exposed to the nursing guide line will be improve.

Instrumentation and tool:

Three tools designed and used by the researcher for collecting data 


\section{Tool one: patient assessment}

This tool developed by the researchers after review of literatures to assess the patient condition to form baseline data it include:

Part I: demographic patient's profile that included the patient's code, age, sex.

Part II: patients clinical data include:

The medical diagnosis ,Date of admission , and Date of discharge from ICU.

Part III: assessment of hemodynamic status which included respiratory system(respiratory rate, spo2) and cardiovascular system (heart rate, blood pressure, systolic and diastolic blood pressure and mean arital blood pressure(three measurement per day).

Part IV: Assessment of the MV parameter which included: mode of ventilation, tidal volume vt, fraction of inspired oxygen (fio2), positive end expiratory pressure PEEP. Minute ventilation pressure support (one type per day).

Part V: laboratory test including complete blood picture (Hemoglobin, Hematocrit, RBCs, WBCs and Platelets), blood culture and arterial blood gases.

Tool 2: Patient's outcomes assessment:

This tool include:

Respiratory Infection ,Complications, Length of ICU stay ,and Mortality rate.

\section{Procedures and data collection}

\section{A) Preparatory phase:}

Ethical considerations

- Informed consent was taken from the head of pediatric intensive care unit to carry out this study after explanation the aim and the natural of the study.

- The researcher based on the relevant literature reviewing developed the tool I, II.

- The developed tools were tested for content related validity by selected juries of six critical care medical and three critical care nursing professionals) to asses adequacy of items of the tools.

- A pilot study: carried out on number of five patients to test the applicability of the tools appropriate study modification was done prior to data collection for the actual study pilot study is excluded the sampling.

The tools that developed by the researcher were tested for reliability by cranchs, Alpha and reliability level were (tool one 0.85 , tool two 0.82 )

- and validity level (tool one 0.921 , tool two 0.905 ) which were acceptable to assess the consistency and stability of the tools.

\section{Statistical analysis}

- The data entry and data analysis were done using spss version (19).
- Data were presented as number, percentage, mean standard deviation.

- Chi - quare test was used to compare between qualitative variables.

- Spearman correlation was done to measure correlation between quantitative variables.

- P- value considered statistically significant when $\mathrm{P}$ $<0,05$.

\section{B) Intervention phase: For Both group}

- Assessment vital signs three time per day for seven days by using tool I.

- Assessment of mechanical ventilation parameters once per day for seven days by using tool1.

- Assessment of laboratory test result once per day for seven days by using tooll it include complete blood picture,bloode culture and ABG.

- Assessment of complications once per day or seven days by using tool II it include( hypoxeimea ,dysrhythmia ,bradycardia ,hypotention bronchospasm ,bleeding and respiratory infection .)

C) Evaluation phase

Each patient evaluated three times to measure the effect of nursing guide line of closed suction system regarding patients out comes of the both groups by using tool I and II. 


\section{Results}

Table (1): Frequency distribution of studied patient's regarding socio-demographic characteristics and clinical data (no: 60).

\begin{tabular}{|c|c|c|c|c|c|}
\hline \multirow{2}{*}{ Items } & \multicolumn{2}{|c|}{ Study(30) } & \multicolumn{2}{|c|}{ Control(30) } & \multirow{2}{*}{ P. value } \\
\hline & No. & $\%$ & No & $\%$ & \\
\hline \multicolumn{6}{|l|}{ Age } \\
\hline Mean $\pm \mathrm{SD}($ range $)$ & \multicolumn{2}{|c|}{$5.69 \pm 5.93(10-16)$} & \multicolumn{2}{|c|}{$3.73 \pm 4.87(10-15)$} & 0.170 \\
\hline \multicolumn{6}{|l|}{ Gender } \\
\hline Male & 19 & 63.3 & 17 & 56.7 & \multirow{2}{*}{0.396} \\
\hline Female & 11 & 36.7 & 13 & 43.3 & \\
\hline \multicolumn{6}{|l|}{ Diagnosis } \\
\hline Respiratory Causes & 12 & 40.0 & 11 & 36.7 & \multirow{6}{*}{0.207} \\
\hline cardiac Causes & 2 & 6.7 & 0 & 0.0 & \\
\hline CNS causes & 9 & 30.0 & 11 & 36.7 & \\
\hline GIT causes & 3 & 10.0 & 4 & 13.3 & \\
\hline Renal Causes & 4 & 13.3 & 1 & 3.3 & \\
\hline Other & 0 & 0.0 & 3 & 10.0 & \\
\hline
\end{tabular}

Chi-square test, independent T- test

Table (2): Comparison between the two groups related to vital signs at $1^{\text {st }}, 4^{\text {th }}$, and $7^{\text {th }}$ days no(60).

\begin{tabular}{|c|c|c|c|c|c|}
\hline Variable & Days & Study & Control & $\mathbf{t}$ & P. value \\
\hline \multirow{3}{*}{ Pulse } & $1^{\text {st }}$ dav & $13317+2591$ & $13659+3059$ & -0.467 & 0642 \\
\hline & $4^{\text {th }}$ day & $126.63 \pm 17.82$ & $139.37 \pm 23.46$ & -2.368 & $0.021^{*}$ \\
\hline & $7^{\text {th }}$ day & $124.18 \pm 27.48$ & $143.41 \pm 26.82$ & -2.743 & $0.008 * *$ \\
\hline \multicolumn{6}{|l|}{ Blood pressure } \\
\hline \multirow{4}{*}{$\begin{array}{c}\text { Systolic Blood } \\
\text { pressure }\end{array}$} & & & & & \\
\hline & $1^{\text {st }}$ day & $97.36 \pm 22.01$ & $88.56 \pm 16.3$ & 1.760 & 0.084 \\
\hline & $4^{\text {th }}$ day & $100 \pm 20.57$ & $92.83 \pm 19.53$ & 1.384 & 0.172 \\
\hline & $7^{\text {th }}$ day & $101.31 \pm 20.39$ & $89.87 \pm 18.99$ & 2.250 & $0.028^{*}$ \\
\hline \multirow{3}{*}{$\begin{array}{l}\text { Diastolic Blood } \\
\text { pressure }\end{array}$} & $1^{\text {st }}$ day & $63.67 \pm 15.37$ & $55.89 \pm 11.99$ & 2.185 & $0.033^{*}$ \\
\hline & $4^{\text {th }}$ day & $64.5 \pm 14.84$ & $59.17 \pm 13.69$ & 1.447 & 0.153 \\
\hline & $7^{\text {th }}$ day & $65.78 \pm 15.44$ & $57.33 \pm 12.88$ & 2.301 & $0.025^{*}$ \\
\hline \multirow{3}{*}{$\begin{array}{c}\text { Mean Arterial } \\
\text { Pressure }\end{array}$} & $1^{\text {st }}$ day & $35.11 \pm 9.38$ & $33 \pm 6.02$ & 1.037 & 0.304 \\
\hline & $4^{\text {th }}$ day & $35.83 \pm 8.03$ & $33.78 \pm 9.5$ & 0.905 & 0.369 \\
\hline & $7^{\text {th }}$ day & $36.5 \pm 8.1$ & $33.48 \pm 7.95$ & 1.458 & 0.150 \\
\hline \multirow{3}{*}{ SPO2_ } & $1^{\text {st }}$ day & $94.59 \pm 10.18$ & $95.23 \pm 4.85$ & -0.313 & 0.755 \\
\hline & $4^{\text {th }}$ day & $96.01 \pm 5.29$ & $94.71 \pm 5.6$ & 0.924 & 0.359 \\
\hline & $7^{\text {th }}$ day & $96.1 \pm 5.91$ & $95.16 \pm 7.38$ & 0.547 & 0.586 \\
\hline \multirow{4}{*}{ Temperature } & & & & & \\
\hline & $1^{\text {st }}$ day & $36.62 \pm 1.07$ & $36.68 \pm 0.93$ & -0.217 & 0.829 \\
\hline & $4^{\text {th }}$ day & $36.78 \pm 0.43$ & $36.47 \pm 0.59$ & 2.342 & $0.023 *$ \\
\hline & $7^{\text {th }}$ day & $36.76 \pm 0.59$ & $36.68 \pm 0.56$ & 0.499 & 0.619 \\
\hline
\end{tabular}

independent T- test $*$ Significant difference at p. value $<0.05$, ** Significant difference at p. value $<0.01$ 
Table (3):Comparison between the two groups related to MV parameters variables at $1^{\text {st }}, 4^{\text {th }}$, and $7^{\text {th }}$ days of admission.

\begin{tabular}{|c|c|c|c|c|c|}
\hline Items & Days & Study & Control & $\mathbf{t}$ & P. value \\
\hline \multirow{3}{*}{$\begin{array}{l}\text { Mandatory } \\
\text { respiration }\end{array}$} & $1^{\text {st }}$ day & $23.53 \pm 8.31$ & $24.53 \pm 8.61$ & -0.458 & 0.649 \\
\hline & $4^{\text {th }}$ day & $22.33 \pm 8.16$ & $27.97 \pm 10.2$ & -2.362 & $0.022 *$ \\
\hline & $7^{\text {th }}$ day & $21.67 \pm 7.62$ & $26.23 \pm 10.83$ & -1.889 & 0.064 \\
\hline \multirow{3}{*}{$\begin{array}{l}\text { Spontaneous } \\
\text { Respiration }\end{array}$} & $1^{\text {st }}$ day & $3481+100$ & $3630+1223$ & 0528 & 0600 \\
\hline & $4^{\text {th }}$ day & $38.13 \pm 10.56$ & $32.76 \pm 9.65$ & $\begin{array}{r}-0.5<0 \\
-2.056\end{array}$ & $0.044 *$ \\
\hline & $7^{\text {th }}$ day & $39.17 \pm 8.6$ & $33.76 \pm 10.29$ & -2.210 & $0.031 *$ \\
\hline \multirow{4}{*}{ VT } & $1^{\text {st }} 1^{2}$ & & & & \\
\hline & $1^{\text {st day }}$ & $116.57 \pm 102.07$ & $69.83 \pm 67.56$ & 2.091 & $0.041 *$ \\
\hline & $4^{\text {th }}$ day & $114.53 \pm 104.76$ & $72.9 \pm 81.34$ & 1.719 & 0.091 \\
\hline & $7^{\text {th }}$ day & $107.63 \pm 99.52$ & $72.63 \pm 95.62$ & 1.389 & 0.170 \\
\hline \multirow{4}{*}{ Fio2 } & & & & & \\
\hline & $1^{\text {st }}$ day & $66.37 \pm 23.96$ & $81.6 \pm 18.03$ & -2.783 & $0.007 * *$ \\
\hline & $4^{\text {th }}$ day & $64.67 \pm 20.87$ & $64.97 \pm 19.13$ & -0.058 & 0.954 \\
\hline & $7^{\text {th }}$ day & $68 \pm 23.86$ & $68.33 \pm 20.95$ & -0.057 & 0.954 \\
\hline \multirow{4}{*}{ PIP } & & & & & \\
\hline & $1^{\text {st day }}$ & $14.13 \pm 3.44$ & $15.2 \pm 2.99$ & -1.282 & 0.205 \\
\hline & $4^{\text {th }}$ day & $15.07 \pm 4.2$ & $15.5 \pm 4.35$ & -0.392 & 0.696 \\
\hline & $7^{\text {th }}$ day & $14.83 \pm 4.34$ & $15.47 \pm 4.03$ & -0.586 & 0.560 \\
\hline \multirow{3}{*}{ PEEP } & $1^{\text {st }}$ day & $5.48 \pm 2.55$ & $4.53 \pm 1.05$ & 1.886 & 0.064 \\
\hline & $4^{\text {th }}$ day & $5.48 \pm 1.9$ & $5.03 \pm 1.9$ & 0.918 & 0.363 \\
\hline & $7^{\text {th }}$ day & $5.32 \pm 1.74$ & $4.98 \pm 1.46$ & 0.818 & 0.417 \\
\hline
\end{tabular}

Table (4): Comparison between the two groups related to mode of mechanical ventilation at 1st, 4th, and 7 th days of admission.

\begin{tabular}{|c|c|c|c|c|c|c|}
\hline Mode $1^{\text {st }}$ day & & & & & & \\
\hline Cpap & 1 & 3.3 & 0 & 0.0 & \multirow{3}{*}{1.154} & \multirow{3}{*}{0.562} \\
\hline SIMV & 9 & 30.0 & 8 & 26.7 & & \\
\hline $\mathrm{A} / \mathrm{C}$ & 20 & 66.7 & 22 & 73.3 & & \\
\hline \multicolumn{7}{|l|}{ Mode $4^{\text {th }}$ day } \\
\hline Cpap & 1 & 3.3 & 0 & 0.0 & \multirow{4}{*}{2.654} & \multirow{4}{*}{0.448} \\
\hline SIMV & 14 & 46.7 & 18 & 60.0 & & \\
\hline $\mathrm{A} / \mathrm{C}$ & 14 & 46.7 & 12 & 40.0 & & \\
\hline Spontous & 1 & 3.3 & 0 & 0.0 & & \\
\hline \multicolumn{7}{|l|}{ Mode $7^{\text {th }}$ day } \\
\hline Cpap & 1 & 3.3 & 0 & 0.0 & \multirow{3}{*}{3.104} & \multirow{3}{*}{0.376} \\
\hline SIMV & 16 & 53.3 & 21 & 70.0 & & \\
\hline$\overline{\mathrm{A} / \mathrm{C}}$ & 13 & 43.3 & 9 & 30.0 & & \\
\hline
\end{tabular}

Chi-square test, independent T-test * Significant difference at p. value $<0.05$,

** Significant difference at p. value $<0.01$ 
Table (5): Comparison between the two groups in relation to arterial blood gases.

\begin{tabular}{|c|c|c|c|c|c|}
\hline Items & Days & Study & Control & $\mathbf{t}$ & P. value \\
\hline \multirow{3}{*}{ PH } & $1^{\text {st }}$ day & $7.46 \pm 0.11$ & $7.44 \pm 0.19$ & 0.402 & 0.689 \\
\hline & $4^{\text {th }}$ day & $7.41 \pm 0.15$ & $7.44 \pm 0.23$ & -0.638 & 0.526 \\
\hline & $7^{\text {th }}$ day & $7.39 \pm 0.14$ & $7.44 \pm 0.19$ & -1.172 & 0.246 \\
\hline \multirow{4}{*}{$\mathbf{P a o}_{2}$} & $1^{\text {st }} \mathrm{day}$ & $11808+4808$ & $11471+580$ & 0308 & 0750 \\
\hline & 1 day & $118.90 \pm 48.08$ & $114.11 \pm 58.9$ & 0.508 & 0.159 \\
\hline & $4^{\text {th }}$ day & $104.85 \pm 40.81$ & $92.54 \pm 35.82$ & 1.242 & 0.219 \\
\hline & $7^{\text {th }}$ day & $102.84 \pm 42.65$ & $86.52 \pm 42.37$ & 1.487 & 0.142 \\
\hline \multirow{4}{*}{$\mathrm{PaCO}_{2}$} & $1^{\text {st }}$ do & 40609006 & & $\rho \cap 0$ & 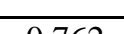 \\
\hline & 1 day & $40.02 \pm 30.04$ & $38.54 \pm 21.38$ & 0.304 & 0.762 \\
\hline & $\frac{4^{\text {th }} \text { day }}{7^{\text {th }} \text { day }}$ & $32.87 \pm 13.23$ & $\frac{52.76 \pm 35.64}{5187 \pm 3276}$ & $\frac{-2.866}{2567}$ & $\frac{0.006 * *}{0013 *}$ \\
\hline & $7^{\text {int }}$ day & $34.84 \pm 15.71$ & $51.87 \pm 32.16$ & -2.567 & $0.013^{*}$ \\
\hline \multirow{3}{*}{$\mathrm{HCO}_{3}$} & $1^{\text {st }}$ day & $24.07 \pm 8.09$ & $26.86 \pm 11.51$ & -1.087 & 0.282 \\
\hline & $4^{\text {th }}$ day & $29.79 \pm 32.87$ & $35.02 \pm 28.28$ & -0.660 & 0.512 \\
\hline & $7^{\text {th }}$ day & $26.72 \pm 7.54$ & $30.95 \pm 12.22$ & -1.614 & 0.112 \\
\hline \multirow{3}{*}{$\mathrm{SaO2}$} & $1^{\text {st }}$ day & $95.53 \pm 7.78$ & $95.57 \pm 4.57$ & -0.022 & 0.982 \\
\hline & $4^{\text {th }}$ day & $95.93 \pm 6.78$ & $96 \pm 2.58$ & -0.053 & 0.958 \\
\hline & $7^{\text {th }}$ day & $96.07 \pm 6.72$ & $96.49 \pm 2.28$ & -0.329 & 0.743 \\
\hline
\end{tabular}

* Significant difference at $p$. value $<0.05$,

** Significant difference at $p$. value $<0.01$

Table (6): Comparison between study and control as regards diagnostic procedures and laboratory investigation.

\begin{tabular}{|l|c|c|c|c|c|c|}
\hline \multicolumn{1}{|c|}{ CBC } & \multicolumn{2}{c|}{ Study } & \multicolumn{2}{c|}{ Control } & t & P. value \\
\hline \hline WBCs1 & \multicolumn{2}{|c|}{$13.17 \pm 5.01$} & \multicolumn{2}{c|}{$17.01 \pm 9.01$} & -2.04 & $0.046^{*}$ \\
\hline culture & No. & $\%$ & No. & $\%$ & & \\
\cline { 1 - 4 } Negative & 22 & 73.3 & 10 & 33.3 & \multirow{2}{*}{$\mathbf{X}^{\mathbf{2}}=9.643$} & \multirow{2}{*}{$0.002^{* *}$} \\
\hline Positive & 8 & 26.7 & 20 & 66.7 & & \\
\hline
\end{tabular}

Table (7): Comparison between study and control regards length of hospital stay.

\begin{tabular}{|c|c|c|c|c|c|c|}
\hline & \multicolumn{2}{|c|}{ Study } & \multicolumn{2}{|c|}{ Control } & \multirow{2}{*}{$\mathrm{X} 2$} & \multirow{2}{*}{ P. value } \\
\hline & No. & $\%$ & No. & $\%$ & & \\
\hline Mortality & 20 & 66.7 & 28 & 93.3 & 6.667 & $0.010^{*}$ \\
\hline \multicolumn{7}{|c|}{ Length hospital stay } \\
\hline 5-10 days & 13 & 43.3 & 14 & 46.7 & \multirow{3}{*}{0.418} & \multirow{3}{*}{0.811} \\
\hline 10-15 days & 7 & 23.3 & 5 & 16.7 & & \\
\hline More than 15 days & 10 & 33.3 & 11 & 36.7 & & \\
\hline Mean \pm SD(range $)$ & \multicolumn{2}{|c|}{$13.34 \pm 7.02(5-32)$} & \multicolumn{2}{|c|}{$14.83 \pm 8.22(5-32)$} & $\mathrm{T}=-0.739$ & 0.463 \\
\hline
\end{tabular}

Chi-square test, $\quad$ * Significant difference at $p$. value $<0.05$,

** Significant difference at p. value $<0.01$

Table (8): Comparison between the two groups regarding to complications from first to seven day.

\begin{tabular}{|l|c|c|c|c|c|}
\hline \multirow{2}{*}{\multicolumn{1}{|c|}{ Items }} & \multicolumn{2}{c|}{ Study } & \multicolumn{2}{c|}{ Control } & \multirow{2}{*}{ P. value } \\
\cline { 2 - 6 } & No & \% & No & \% & \\
\hline hypoxemia & 18 & 60 & 24 & 80 & 0.159 \\
\hline dysrhythmias & 16 & 53.3 & 20 & 66.7 & 0.426 \\
\hline bradycardia1 & 0 & 0 & 0 & 0 & - \\
\hline
\end{tabular}




\begin{tabular}{|l|c|c|c|c|c|}
\hline \multirow{2}{*}{ Items } & \multicolumn{2}{|c|}{ Study } & \multicolumn{2}{c|}{ Control } & \multirow{2}{*}{ P. value } \\
\cline { 2 - 5 } & No & \% & No & \% & \\
\hline hypotension1 & 5 & 16.7 & 3 & 10 & 0.701 \\
\hline bronchospasm1 & 13 & 43.3 & 13 & 43.3 & 0.701 \\
\hline Atelectasis1 & 8 & 26.7 & 15 & 50 & 0.111 \\
\hline trauma1 & 0 & 0 & 0 & 0 & - \\
\hline bleeding1 & 0 & 0 & 1 & 3.3 & 0.999 \\
\hline Respiratory infection & 8 & 26.7 & 20 & 66.7 & $0.004 * *$ \\
\hline Cardic1 & 5 & 16.7 & 2 & 6.7 & 0.421 \\
\hline Respiatory1 & 6 & 20 & 3 & 10 & 0.469 \\
\hline
\end{tabular}

Chi-square test, * Significant difference at $p$. value $<0.05$,

** Significant difference at $p$. value $<0.01$

Table (1): Shows the frequency distribution of the study and the control groups regarding sociodemographic characteristics and clinical data:-

Regarding to age the results of the current study revealed that the mean age of study and control groups were $(5.69 \pm 5.93(10 \quad-16))$ versus $(3.73 \pm 4.87(10-15))$ respectively.

As regarding to sex, it was noticed that more than half of patients in the study group were male patient $(19(63.3 \%))$ versus $(17(65.7 \%)$ respectively and low percent of patients of study and control group were female $(11(36.7 \%)$ versus $(13(43.3 \%)$ respectively.

Regarding to medical diagnosis, results revealed a relatively more than half of patient in the study and control group were having respiratory causes (12(40 $\%)$ versus $(11(36.7 \%)$.

Finally there was no statically significant difference between the two groups (p. value >0.05)

Table (2): Comparison between the two groups related to vital signs at the first fourth and seven days. This table showed that there was statistically significance difference between the two groups regarding temperature, pulse, blood pressure ,spo2 through the first to seven days.

Table (3) \& Table (4): Shows comparison between the two groups related to M.V parameters. This table showed that there was no statistically significance between the two groups regarding most of mechanical ventilation parameters at the first, fourth, seven day of admission (P.value $<0.05$ ).

Table (5): Shows comparison between the two groups in relation to arterial blood gases. This table showed that there was no statistically significant difference between the two groups regarding almost of items of arterial blood gases. (P.value $>0.05$ ).

Table (6): Shows comparison between the two groups in relation to diagnostic procedures and laboratory investigation. There was a statistically significant difference between the two groups regarding almost of diagnostic and laboratory investigation at the first to seven day of admission. (P. value <0.05).

Table (7): Shows comparison between study and control regards outcomes. Concerning to ICU stay there is was no statically significant difference between study and control groups (13.34 \pm 7.02$)$ versus (14.83 \pm 8.22$)$ respectively.

Concerning to mortality rate there was a highly significant increase in the mortality in the control group versus the study group. There was a statistically significance between the two groups (P.value<0.05).

Table (8): Shows comparison between the two groups in relation to complication

There was a significant difference $(\mathrm{P}$. value $<0.05)$ in the control group for respiratory infection more than study group ( $\mathrm{P}$. value $>0.05)$.

\section{Discussion}

This study was done on 60 patients who were mechanically ventilated condition by the closed suction system.To perform the procedure as needed other hand, several criteria describe the potential indication for ETS such as raised respiratory rate, reduced SPO2 levels. (Endocott, 2014), skin color pattern of respiration coughing increased work of breathing (AARC clinical practice guidelines, 2011).

Nurses are with patient at time of admission and take care of patients through their hospital stay. They are in the ideal position to assess patient risk factors early critical care nurses must understand the risk factors of CTS and the various methodologies used to prevent this potentially life. Threating condition, therefore, the current study as carried out to evaluate the effect of implementing nursing guidelines on the procedure of CITS. In critically ill patients since implementing. These guidelines will improve nursing practice and facilitate the utilization of collaborative approach. Providing optimal patients care and improve the clinical outcomes (Hazinskim, 2013). 
One of the advantages of closed suction system is to reduce respiratory pollution and pulmonary infection, Another potential advantage is it east application which only needs one nurse in closed suction method nurse would not be infected by patients endotracheal tube secretions and suction catheter can be used frequently (Elmonsoury, et al., 2016).

Socio-demographic characteristic of studied subject.

Regarding to patient age, sex of the study sample, the findings of the current study revealed that was no statistically significance difference between the two groups our result are supported with (Gillies, 2013) Study which also showed no statistically significance difference between two groups.

Regarding to patient's diagnosis of the study sample the findings of the current study revealed that there was no statistically significance difference between the two groups our results are supported with (Combest, et al., 2016) Study which also showed no statistically significance difference between the study groups compared to control group. Regarding to patients hemodynamic status (heartrate)mean of the study sample the finding of the current study revealed that there was no statistically significance difference between the two groups in seven day, the presence study that the mean of heart rate respiration changed immediately after tracheal suction and reached base line 5minutes. Post suction (Jongerdent, 2014, Ozzden, 2014) which also showed significant increase in mean score of heart rate and respiratory rate were observed after 5 minutes to suction.

Regarding to systolic, diastolic blood pressure of the study sample of the findings of the current study revealed that there was statistically significant difference between two groups in the seven day our results are supported with (Yazdonnik, 2013) Show a significant in hemodynamic status. Regarding to Arterial blood oxygen saturation SPO2 of patients increase $d$ after suction inboth group, there were no statistically significant difference between two groups our result are supported with(Evans, 2014).

Regarding to mechanical ventilation parameters of the study sample, the finding of the current study revealed that there was o statistically significant difference between the two groups regarding the most of mechanical ventilation parameters our results are contradicted with (Royal, 2012).Study which also showed statistically significant, close d suction can also maintain continuous ventilation and positive end-respiratory pressure(PEEP)to avoid are duction alveolar atrophy.(Curell, 2012).

Regarding to arterial blood gases of the study sample, the finding of the current revealed that there was no statistically significance difference between two groups in spit of into group but improvement in study group due to small number of patient our result are supported with (Jondern et al., 2012) Study which also showed statistically significant difference between the study groups compared to control group. This finding result can be interpreted that there was effect of multiple factors effects on results as progress of disease and complications.

Regarding to diagnostic procedure and laboratory investigation of the study sample the finding of the current study revealed that there was statistically significant difference between the two groups our results are supported with (Sharma, 2014). Which also showed statistically significant difference between the study group compared to control group increase negative blood culture 22 more than fifty percent In the study group 10 less than fifty percent because some nurses did not wear goggles should be observe wearing gloves during ETS, should be maintain aseptic technique s including hand washing before and after suction.

Regarding to length of stay (days) of the study sample, the finding of the current study revealed that was no statistically significant difference between the s tudy and control groups ( 13.34 $\pm 7.02(5-32)$ versus $(14.83 \pm 8.22(5-32)$ o u r result are supported with (Topleil et al., 2012), study which also showed no statistically significant difference between the two group.

Regarding to mortality rate of the study sample the finding of the current study revealed that there was significant between two group increases in the control groups than study group our result are supported with (Jeong, 2014) Study also showed statistically significant difference between the two groups.

Regarding to complication occurrence of infection of the study sample the finding of the current study revealed there was statistically significant difference between the two groups regarding complication. Hypoxemia, dysrhythmia were increased in the control group than study group. Hypoxemia leads to hemodynamic changes when removing oxygenated airway along of time with the bronchial secretion during closed suction. Other hands, hyper oxygenation prior to and after suctioning respectively. However, ventilation and oxygenation during CSS continuous. Although the routine $(\mathrm{NaCl})$ instillation is not recommended for closed suction his subject still failed (Yazidim, 2013).

\section{Conclusion}

This study evaluated the effect of nursing guideline of closed suction on patient's outcomes there is no different between two groups related to the 
application of nursing guideline of closed suction regarding was improvement outcomes in the study group than control group.

\section{Recommendation}

This study recommended that keep the nursing guideline of closed suction system available in all the intensive care unit, in generalized providing training programs for new joined ICU nurse about nursing guideline of closed suction system in the future. Reapplication of the study on a large probability of sample from different ICU is required.

\section{References}

1. Elsaman S., (2017): Effect of Application of Endotracheal Suction Guidelines on Cardiorespiratory Parameters of Mechanically Ventilated Patients. IOSR Journal of Nursing and Health Science. Volume 6, Issue 1 Ver. I (Jan. - Feb. 2017), PP 41-48.

2. Khimani1 R., (2015): Practices of Tracheal Suctioning Technique among Health Care Professionals: Literature Review International Journal of Nursing Education., Vol. 7, No.1,PP 30-33

3. Sharma S., (2014): Effectiveness of "endotracheal suctioning protocol" in terms of knowledge and practices of nursing personnel. Nursing and Midwifery Research Journal, Vol10, No.2 PP42-48.

4. Hazinski, M., (2013): Nursing Care of the Critically Ill Child, 3rd Edition. Elsevier Mosby: St. Louis ,PP302-330

5. Endacott R., (2014): Tracheal and endotracheal stationary in: Endacott R, editor. Clinical nursing skills core and advanced. $1^{\text {st }}$ ed USA: oxford University press;. Pp. 381-612.

6. AARC clinical practice guidelines endotracheal suctioning of mechanically ventilated patient with artifial airways (2011).

7. Dougherty, L., (2015): The Royal Marsden Hospital Manual of Clinical Procedures. 9th Edition. John-Wiley: Chichester PP202:250.

8. Gillies, D., (2013): Deep Versus Shallow Suction of Endotracheal Tubes in Ventilated Neonates and Young Infants. Cochrane Database of systematic Reviews Aaliable online:

http//www.cochranelibrary.com/(Accessed October $27^{\text {th }} 2015$ ).

9. Lippincot, Williams and Wilkinson (2011) Show me the Evidence.

10. http//www.

Nursingcenter.com/evidencebasedpracticenetwo rk/home/tools- resources/collections/understandingevidencebas edpractice.aspx(Accessed February $28^{\text {th }}$ 2013).

11. Royal Children's Hospital (2012): Endotracheal Suctioning of Ventilated Neonates. RCH: Melbourne. Available online:

12. http://www.rch.org.au/rchcpg/hospital_clinical_ guideline_index/Endotracheal_Tube_suction_of _Ventilated_Neonates/(Accessed October $21^{\text {st }}$ 2015)

13. Cullen L., (2012): Planning for implementation of evidence-based practice. ,PP 22:30.

14. Tropeil A., (2014): Comparison of the effect of closed versus open endotracheal suction systems on the development of ventilator-associated pneumonia. J Hosp Infect.;58(1):14-9.

15. Combes P., (2016): Nosocomial Pneumonia in mechanically ventilated patients, a prospective randomized evaluation of the stericath closed suctioning system. Intensive care Med. ;26(7):878-82.

16. Yazdonnik A., (2013): Comparing two levels of closed system suction pressure in ICU patients: Evaluating the relative safety of higher values of suction pressure. Iran $\mathbf{J}$ Nurs Midwifery Res.;18(2):117-22.

17. Evans J., (2014): comparison of open and closed sucion on safty, efficiency and nursing time in a pediatric intensive care unit. Aust critcare.; 27 (2): 70-4 (Do) (pumped)

18. Frota O., (2014): open system endotracheal suctioning: practices of intensive care nursing professionals. 18 (2): 296 - 302.

19. Jeong J., (2014): A closed - suction catheter with a pressure valve can Reduce tracheal mucosal injury in intubated patients korean $\mathrm{j}$ crit care $-29(1): 7-12$.

20. Elmansoury A., (2016): closed suction system versus open suction Egyptian Journal of chest Diseases and tuberculosis, PP50-56

21. Jongerden I., (2012): changes in heart rate ,mean arterial pressure,and oxygen saturation after open and closed endotracheal suctioning :Aprospective observational study.Journal of critical care.Article in press.

22. Ozden D., (2014): Nursing in critical published by John wiley and sons. Vol (20), No 3. 